\title{
Desregulación emocional, rumiación e ideación suicida en estudiantes que cursan estudios generales en una universidad pública de Lima Metropolitana
}

\author{
Emotional disregulation, rumiation and suicide ideation in students of \\ general studies of a university Metropolitan Lima public
}

\author{
Carlos Velásquez Centeno ${ }^{1}$, Alex Grajeda Montalvo 2, Víctor Montero López ${ }^{3}$, \\ William Montgomery Urday ${ }^{4}$ \\ Universidad Nacional Mayor de San Marcos \\ Kevin Egusquiza Vásquez ${ }^{5}$ \\ Instituto Nacional de Salud Mental Honorio Delgado Hideyo Noguchi
}

Recibido: $25-05-20$

Aceptado: $12-06-20$

\section{Resumen}

La presente investigación aborda las relaciones entre la desregulación emocional, la rumiación y la ideación suicida. El estudio comprende primeramente la elucidación de los conceptos teóricos de las variables implicadas, entendiéndolas bajo un marco paradigmático de referencia llamado "contextualismo funcional". Respecto al método, las variables se miden por medio de las Escalas de Dificultades de la Regulación Emocional, de Respuestas Rumiativas y de Ideación Suicida Positiva y Negativa, aplicándose todas a una muestra de 1330 estudiantes de la Escuela de Estudios Generales de una Universidad Pública de Lima, matriculados en el año Académico 2018. Los resultados muestran que sí existen correlaciones significativas entre desregulación emocional, rumiación e ideación suicida, siendo la mayor la encontrada entre desregulación emocional y rumiación. En particular, se ve también que los ingresantes al área de humanidades son en promedio más vulnerables a padecerlas.

\footnotetext{
${ }^{1}$ Docente, Universidad Nacional Mayor de San Marcos. Lima, Perú. E-mail: cvelasquezc@unmsm.edu.pe. ORCID: https://orcid.org/0000-0002-7266-4012

${ }^{2}$ Docente, Universidad Nacional Mayor de San Marcos. Lima, Perú. E-mail: agrajedam@unmsm.edu.pe. ORCID: https://orcid.org/0000-0001-5972-2639. Autor para correspondencia.

${ }_{3}^{3}$ Docente, Universidad Nacional Mayor de San Marcos. Lima, Perú. E-mail: vmonterol@unmsm.edu.pe. ORCID: https://orcid.org/0000-0002-9968-4160

${ }^{4}$ Docente, Universidad Nacional Mayor de San Marcos. Lima, Perú. E-mail: wmontgomeryu@unmsm.edu.pe, avidolector@,yahoo.es. ORCID: https://orcid.org/0000-0001-9518-3329

${ }^{5}$ Psicólogo, Instituto Nacional de Salud Mental Honorio Delgado Hideyo Noguchi. E-mail: kev9nhev@gmail.com. ORCID: https://orcid.org/0000-0001-6842-4369
}

(C) Los autores. Este artículo es publicado por la Revista de Investigación en Psicología de la Facultad de Psicología, Universidad Nacional Mayor de San Marcos. Este es un artículo de acceso abierto, distribuido bajo los términos de la licencia Creative Commons Atribucion - No Comercia_Compartir Igual 4.0 Internacional. (http://creativecommons.org/licenses/by-nc-sa/4.0/) que permite el uso no comercial, distribución y reproducción en cualquier medio, siempre que la obra original sea debidamente citada. 
Palabras clave: Desregulación emocional; rumiación; ideación suicida; contextualismo; salud psicológica.

\begin{abstract}
In the present investigation, the relationships between emotional dysregulation, rumination and suicidal ideation are addressed. The study first includes the elucidation of the theoretical concepts of the variables involved, understanding them under a reference paradigmatic framework called "functional contextualism". Regarding the method, the variables are measured by means of the Difficulties Scales of Emotional Regulation, Ruminative Responses and Positive and Negative Suicidal Ideation, all applying to a sample of 1330 students from the School of General Studies of a Public University of Lima, enrolled in the Academic year 2018. The results show that there are significant correlations between emotional dysregulation, rumination and suicidal ideation, the highest being found between emotional deregulation and rumination. In particular, it is also seen that those entering the humanities area are on average more vulnerable to suffer them.
\end{abstract}

Keywords: Emotional dysregulation; rumination; suicidal ideation; contextualism; psychological health.

La desregulación emocional es un concepto clínico introducido por la destacada psicoterapeuta Marsha Linehan (2003), el que está relacionado con múltiples desórdenes del comportamiento, especialmente con los trastornos límite, histriónico y de estrés postraumático. En ellos es un factor que implica respuestas emotivas escasamente controladas por parte del individuo afectado, como por ejemplo las que aparecen en accesos intensos de ira o de histeria. La impulsividad es un elemento importante en este tipo de respuestas, que pueden llegar incluso a la autoagresión. De acuerdo con Linehan (2003), en el caso de personas con trastorno de personalidad límite aquellas experimentan un conjunto variable de emociones intensas y poco gratas (por ejemplo ira, frustración, tristeza o ansiedad), mostrándose impotentes para manejarlas, o siquiera comprenderlas en el contexto de una situación determinada. Por otro lado, se supone que el origen del problema de la desregulación emocional se halla asociado al sufrimiento infantil de abusos, malos tratos o negligencia en el cuidado, sea a manos de familiares o de personas vinculadas a instituciones de crianza (Schore, 2003; Gratz \& Roemer, 2004).

Las rumiaciones constituyen un flujo incesante de pensamientos obsesivos sobre la previsión y planificación de cada paso que lleva a cabo el individuo que los experimenta, cuya finalidad, al parecer, es aliviar el sentimiento de ansiedad e inseguridad que sufre tratando de encontrarle un sentido a la situación que vive. Esto, que proporciona una falsa sensación de control y seguridad, sin embargo, no funciona de forma duradera, empeorando en realidad la forma de enfrentamiento con los diversos problemas cotidianos porque la característica principal de la rumiación es el enfoque casi exclusivo no en las soluciones a tales problemas, sino en las posibles causas y consecuencias de su sufrimiento, en los aspectos negativos 
y difíciles. Por ello se entiende que las rumiaciones tienen, por lo general, mucho que ver con estados depresivos (Hervás y Vázquez, 2006).

En cuanto a la ideación suicida, esta comprende una serie de discursos verbales abiertos o encubiertos que pueden involucrar deseos y/o planes de auto-eliminación, a veces exhaustivamente detallados y a veces simplemente formulados a manera de queja. Si bien el rango de expresión de tales discursos es susceptible de variar entre una real intención de acabar con la muerte o solo de "amenazar" con hacerlo para conseguir atención o beneficios; lo cierto es que la mayoría de individuos con ideación suicida no llega a concretar su propósito explícito (Gliatto \& Rai, 1999; Rosales, Córdova y Ramos, 2012). Sin embargo, obviamente la ocurrencia de ideaciones suicidas es, sin duda, un factor de riesgo para la persona, y se asocia tanto a ciertos trastornos del estado de ánimo como a la depresión, sin dejar de lado patologías (bipolaridad, esquizofrenia) o situaciones personales o familiares que influyen en su incidencia. Como en el caso de la desregulación emocional, la ideación suicida también es una respuesta característica del trastorno límite de la personalidad (Linehan, 2003); siendo a menudo consecuencia de la desesperación ligada a la experiencia de disgustos o desgracias personales, de enfermedades o de abuso de sustancias.

Respecto a estos temas existen teorías de orden biológico, social y psicológico que se verán sucintamente a continuación, de acuerdo con la revisión de Villalobos (2009):

Las teorías biológicas, fundamentalmente representadas por el trabajo de Van Heeringer, enfatizan el papel serotosinérgico (influencia de la serotonina en el organismo en conjunción con el sistema noradrenérgico) como factor de riesgo para la predisposición a sufrir problemas vinculados a la falta de cognición prospectiva y poca tolerancia al estrés psicosocial, a déficits en solución de problemas, a la depresión, $\mathrm{y}$, por consiguiente, a la desregulación emocional, las rumiaciones y la ideación suicida.

Las teorías sociales, de las cuales Durkheim es el principal autor representativo, giran en torno al sentido de comunalidad supuestamente vigente en todos los individuos. Vale decir, la interacción armónica que se establece entre el individuo y su entorno social como estructura para su existencia. La desintegración de dicha estructura es la que se relaciona con trastornos emotivos como los reseñados, al amenazar el delicado equilibrio que permite controlarlos. Según esto, las condiciones desintegrativas pueden llevar a que el individuo piense que su muerte es necesaria para el bienestar de su comunidad; y otras veces puede creer que esa misma comunidad es un obstáculo insuperable para su desarrollo personal, no quedando otra salida que escapar mediante el suicidio. 
Las teorías psicológicas son las más numerosas. Aquí solo se reseñan las principales.

La hipótesis del instinto de muerte psicoanalítico alude al sufrimiento melancólico del individuo que identifica al objeto amado consigo mismo, y, al advertir cosas odiosas en él tiende a sentir hostilidad hacia su propia persona. El corolario resulta ser una forma de auto-castigo a través del pensamiento recurrente sobre su auto-eliminación. Esta ira hacia sí mismo puede tener correlatos de insuficiente desarrollo del ego con respecto a los impulsos autodestructivos inherentes a todo ser humano. Otras contribuciones psicodinámicas analizan el fenómeno a través de la hipótesis sobre déficits interpersonales que los individuos tratan de compensar mediante la búsqueda personal de manipulación, de atención o de expresión catártica de la emoción depresiva; o simplemente como una reacción ante el dolor psicológico.

Desde la perspectiva de la teoría cognitiva de la depresión, Beck (2005) supone que existe una relación fundamental con la desesperanza captada a través de una serie de ideas distorsionadas sobre sí mismo, el mundo y el futuro, exagerando sus problemas y considerando la muerte como una forma lógica de solución a ellos. En este proceso pueden existir motivos más o menos graves en un continuo de incidencia. Los más graves se guían por niveles elevados de depresión y desesperanza, mientras que los menos graves están ligados a deseos de manipulación de sentimientos y conductas ajenas. Otras teorías cognitivas enfatizan aún más la variable "desesperanza", convirtiéndola en un concepto cuasiequivalente a la indefensión como actitud fatalista según la cual lo malo le tenderá a pasar sin que haya ningún tipo de posibilidad para detenerlo. La anticipación de fracaso es, pues, un factor importante en esta aproximación.

La teoría del aprendizaje social también reconoce el aspecto de la indefensión, añadiendo que es progresivamente aprendida y socialmente reforzada en etapas tempranas del desarrollo. También se puntualiza que la actitud del individuo hacia el suicidio es, en este contexto, un recurso manipulativo ejercido con fines de producir reacciones sociales. En otros casos, la falta de refuerzos y/o de metas vitales se pueden tornar tan significativas que llevan a la depresión, y con ella, a sus correlatos emocionalmente desregulativos, rumiativos e ideacionales negativos (Bandura, 2002).

Finalmente, el modelo procesual del estrés proveniente de Lazarus y Folkman (ver Lazarus, 1999), da predominancia explicativa a los acontecimientos estresantes. Estos - se dice desde la perspectiva señalada- incrementan la vigilancia del sujeto hacia circunstancias difíciles de la vida, predisponiéndolo a sesgos cognitivos de juicio respecto a ellos y sus consecuencias indeseables. 


\section{Del marco paradigmático de referencia}

La aproximación asumida por los autores del presente estudio se halla inserta en lo que se suele denominar "terapia de conducta de tercera generación". Esto ha sido llamado "contextualismo funcional": un tipo de pragmatismo psicológico que se caracteriza por definir el comportamiento como un acto-en-contexto histórico y situacional (e incluye la cognición como conducta en función). Aunque, como es conocido, rescata planteamientos conductuales, le añade nociones como la aceptación, los valores y la atención plena (Hayes, Villatte, Levin \& Hildebrandt, 2011; Hayes, Levin, Plumb-Vilardaga, Villatte \& Pistorello, 2013).

Desde este enfoque paradigmático se considera, al revés de otras perspectivas, que los pensamientos y sentimientos - así como sus mutuas relaciones- no son "causas" de la acción, como tampoco está lo es de sí misma. Todas ellas funcionan en contextos históricos y situacionales que las propician a través de reglas establecidas por marcos relacionales ${ }^{6}$ de tipo verbal. Los valores, por ejemplo, no son más que consecuencias temporalmente distantes y construidas verbalmente de aquello que se desea en la vida.

Por tanto, como señalan Barraca y Pérez (2015), la cuestión fundamental respecto a los problemas clínicos asociados a la ansiedad y la depresión es cómo llegan a serlo y de dónde vienen. Cuáles son aquellas condiciones-límite que inciden en ellos. A este nivel se pueden considerar los contextos verbal-relacionales como relevantes.

En efecto, tradicionalmente se han visto los problemas de "salud mental" y del sufrimiento como señales de trastorno y enfermedad, lo que configura un paradigma cultural de evitación como señal de "sanidad". En otras palabras, el contexto social-verbal en que vive un individuo le impone como regla que ciertos estados emocionales son anormales y negativos (por ejemplo la desesperanza y el deseo de suicidio), y que debe evitarlos. El problema de esta conceptualización es que lo que se ve en la realidad es que hay una enorme mayoría de personas que atraviesan en alguna etapa de su vida por tales "desarreglos" emotivos, lo cual por definición no puede ser "anormal". En verdad ningún pensamiento, sentimiento o recuerdo es patológico independientemente de la función que cumple en una circunstancia concreta. ¿Cómo puede ser "negativo" per se un proceso de adaptación desarrollado para defenderse de condiciones adversas?

Lo cierto es que solo el ser humano experimenta deseos conscientes de quitarse la vida, porque tiene algo que otros organismos no tienen. Esto es el lenguaje y el contexto relacional. Como dicen Hayes, Strosahl \& Wilson (2014):

\footnotetext{
${ }^{6}$ Para efectos de esta aproximación, "relacionar" es "responder a un evento en términos de otro". Sobre esta base se pueden establecer relaciones de coordinación, temporales y causales, comparativos y evaluativos, deícticos y espaciales (Hayes \& Blackledge, 2013).
} 
"Los seres humanos pueden anticipar sucesos aversivos, crear semejanzas y disimilitudes entre acontecimientos y establecer relaciones entre acontecimientos del pasado y del presente basadas en la elaboración de semejanzas. Los humanos pueden generar predicciones sobre situaciones que todavía no han experimentado, pueden responder como si se estuviera dando una situación aversiva cuando esta ya concluyó décadas atrás" (p. 43). Y todo eso bajo la influencia de contextos verbales que, como se ha dicho en anteriores líneas, condenan el sufrimiento, siendo dicho marco contingencia el verdadero factor de riesgo predisponente a las rumiaciones perfeccionistas y los deseos suicidas.

Cuando un individuo toma como ciertas (y se guía por) las afirmaciones evaluativas de ese marco relacional inadecuado sobre los eventos de la vida y de su yo sin atender a la experiencia sensorial que siente en el presente, se habla de "fusión cognitiva" y de "evitación experiencial", dos procesos que inflexibilidad el comportamiento y lo hacen fácil presa de la depresión. Desregularse emocionalmente y pensar en quitarse la vida son las consecuencias. La rumiación no es más que un intento de control fallido sobre el problema que ha sido creado por la rigidez de creerse el cuento socio-verbal de que hay emociones y reacciones negativas que deben eliminarse a la fuerza para ser "sano". Por eso este enfoque propugna el desmantelamiento de ese contexto de explicación y el retorno a formas contemplativas de la propia experiencia, buscando resituarla como una función en otro contexto de defusión y aceptación del sufrimiento como algo natural (Shaurya, Whitmoyer, Aldao \& Schirda (2017)). El objetivo del presente estudio es proporcionar información previa sobre la población universitaria para preparar estrategias que lleven a esa meta.

\section{ANTECEDENTES}

En la actualidad, se considera al suicidio como un problema de salud pública a nivel internacional que requiere especial atención desde múltiples niveles (OMS, 2003), siendo la segunda causa principal de muerte en población adolescente y adulta joven (OMS, 2015). Esto implica que no sólo se destinen esfuerzos en la búsqueda por lograr mejores resultados a través del tratamiento, sino también en promover medidas oportunas para la detección temprana de la conducta suicida y desplegar estudios que apunten a mejorar la comprensión de los factores psicológicos que se encuentran íntimamente ligados a ésta. En el marco del estudio de la conducta suicida se denomina espectro suicida a los diferentes estadios -de menor a mayor gravedad- en los que es capaz de manifestarse el suicidio. Una de las expresiones más frecuentes y de inicio temprano es la idea suicida, definida como la presencia de pensamientos o deseos de cometer suicidio haciendo uso de un plan e identificando los mecanismos para llevarlo a cabo. Por su parte, la rumiación implica un estilo de respuesta a través del cual las personas centránse en sensaciones tipo la tristeza y sus consecuencias o 
causas, en vez de trazar estrategias de resolución de problemas que auxiliarían positivamente a reducir aquellos eventos privados (Nolen-Hoeksema, 1991). Frente a ello, Pérez-Álvarez acota que la rumiación implica una conducta de analizar reiteradamente sobre un mismo hecho sin que estos sean explicativos de la situación (Pérez-Álvarez, 2014). Velásquez, Grajeda, Montero, Montgomery y Egusquiza (2018) encontraron correlaciones positivas y grandes entre rumiacion e impulsividad y también entre la rumiación y la evitación experiencial en estudiantes universitarios sanmarquinos.

Por otro lado, la desregulación emocional es definida por especialistas del comportamiento clínico como una alta vulnerabilidad para regular las emociones, así como un déficit en la habilidad para modular dichas emociones (Linehan, 2003). En esa línea, se han realizado conceptualizaciones más modernas que sientan las bases de la regulación emocional a la luz de cuatro conceptos base: la selección de situaciones, el despliegue atencional, el cambio cognitivo y la modulación de la respuesta (Seligowski \& Orcutt, 2015). Diversos estudios han analizado la relación entre la evitación experiencial y la rumiación, contiguo a la depresión y ansiedad en poblaciones no clínicas, tal cual el tema de los estudiantes universitarios, en tanto los resultados muestran relaciones significativas entre ambas variables. Así, Berghoff, Pomerantz, Pettibone, Segrist, y Bedwell (2012) evaluaron la relación entre la evitación experiencial y la impulsividad en estudiantes universitarios, ellos encontraron una relación significativa entre ambas variables, tenemos así que la impulsividad de tipo no planeada es la mejor predictora de evitación experiencial. Nicolai (2015) examinó la rumiación e impulsividad en tanto variables mediadoras de conductas de autolesiones no suicidas y la afectividad negativa en estudiantes universitarios. En su investigación encontró que ambas variables operaban como mediadoras de la relación entre conductas de autolesiones no suicidas y la afectividad negativa. A su vez, Serrano y Flores (2005) indagaron sobre estrés, respuestas de afrontamiento e ideación suicida en adolescentes, buscando establecer relaciones entre estas variables, Los resultados indican que a mayor estrés con la pareja, mayor ideación suicida en los hombres. En las mujeres, la ideación suicida se asoció con un mayor estrés social y con la pareja. Asimismo, se encontró correlación moderada, significativa y positiva entre los factores de las escalas de afrontamiento y la ideación suicida. En otro estudio, Suárez, Restrepo y Caballero (2016) consideraron la Ideación suicida y su relación con la inteligencia emocional en estudiantes de Universidades de Colombia. De acuerdo con su argumentación, pensamientos acerca de la muerte e ideas de suicidarse pueden presentarse de manera habitual en estos universitarios durante el semestre académico, debido a las nuevas experiencias de vida en los jóvenes, los cuales se ven enfrentados a significativas presiones sociales, personales, económicas y académicas que algunos estudiantes logran enfrentar de manera efectiva mientras otros no. Los resultados muestran que el sostenimiento de 
ideas suicidas responde a conflictos de éstos jóvenes para percibir sus estados emocionales, reparar emociones negativas y afrontar problemas. Por tanto, la falta de habilidades de inteligencia emocional son factores psicológicos que juegan un rol fundamental en la aparición y mantenimiento de ideas suicidas. Finalmente, de acuerdo con Inostroza (2016), podemos arribar a la conclusión que, la desesperanza, el afrontamiento centrado en la tarea, y el afrontamiento emocional desadaptativo, son procesos psicológicos de riesgo que modulan la influencia del estrés vital y los síntomas depresivos en la tentativa de suicidio del último año en adultos que eran pacientes de salud mental. La ideación suicida tiene un rol notable en tanto factor de riesgo proximal al intento suicida.

Como se ha precisado, las muertes por suicidio ocurren con mayor incidencia entre los 15 y 29 años, lo cual corresponde a las etapas evolutivas de adolescencia tardía y adulta joven, edades en las que por lo general transcurre la formación educativa universitaria. Por ello, el presente tiene como objetivo principal analizar la relación entre la desregulación emocional, la rumiación y la ideación suicida en los estudiantes universitarios que cursan estudios generales en una Universidad Pública de Lima metropolitana.

\section{MÉTODO}

\section{Enfoque tipo y diseño de investigación}

Nuestro estudio posee un enfoque cuantitativo bajo un tipo de investigación descriptiva correlacional y con un diseño no experimental transversal (Sánchez y Reyes, 2015).

\section{Muestra}

Se utilizó una muestra accidental, conformada por 1330 estudiantes de la Escuela de Estudios Generales de una Universidad Pública de Lima Metropolitana. De los cuales 742 son de género masculino y 588 del femenino, con edades que oscilan entre 16 a 31 años los cuales atañen a cinco Aéreas: Ciencia de la Salud (337), Ciencias Básicas (153), Ingeniera (353), Ciencias Económicas y de la Gestión (204) y Humanidades y Ciencias Jurídicas y Sociales (283) registrados en el año Académico 2018.

Variables e instrumentos:

Los siguientes instrumentos sirvieron para medir las variables estudiadas:

- Desregulación Emocional, mediante la Escala de Dificultades de la Regulación Emocional (DERS-E; Hervás y Jódar, 2008).

- Rumiación, mediante la Escala de Respuestas Rumiativas (RRS; Hervás, 2008). 
- Ideación Suicida, mediante la Escala de Ideación Suicida Positiva y Negativa (PANSI; Villalobos, 2009, 2010).

- La edad, sexo y áreas profesionales, fueron las variables socio demográficas.

Estudio de la Confiabilidad y Validez en instrumentos empleados: "Los instrumentos de medición psicológica, al igual que otros instrumentos de medición en otras ciencias, deben de poseer ciertas características que aseguren, en cierta medida, la veracidad y confianza de los resultados obtenidos" Grajeda (2018) p.23. Por dicho motivo se estudió la Valdez y confiabilidad instrumental que a continuación se detalla:

Realizamos un estudio psicométrico de la Escala de Dificultades de la Regulación Emocional con las puntuaciones obtenidas encontrando que la prueba posee validez de constructo ya que las correlaciones ítem test oscilaron entre 0.29 y 0.78 . De otro lado la confiabilidad determinada por el Alpha fue igual a 0.94 siendo muy alta.

Nuestro estudio psicométrico de la Escala de Respuestas Rumiativas, encontró que la prueba posee validez de constructo ya que las correlaciones ítem test oscilaron entre 0.26 , (salvo el ítem 12 que obtuvo 0,16 ) y 0.528 . De otro lado la confiabilidad determinada por el Alpha fue igual a 0.92 siendo muy alta.

Finalmente el estudio psicométrico la Escala de Ideación Suicida Positiva y Negativa con las puntuaciones obtenidas determinó que la prueba posee validez de constructo ya que las correlaciones ítem test oscilaron entre 0.334 y 0.841 . De otro lado la confiabilidad determinada por el Alpha fue igual a 0.80 siendo alta.

\section{Procedimiento:}

La aplicación de los instrumentos a los estudiantes se concretó en coordinación y con el consentimiento de docentes y autoridades de una Universidad Pública de Lima Metropolitana, en los días y horas programados, en forma voluntaria y en sus ambientes naturales.

Los datos obtenidos fueron procesados usando el Programa SPSS (versión 24), usando estadística descriptiva al caracterizar la variable de estudio; así como estadística inferencial al establecer la diferencias conformes a la normatividad de la curva, y el análisis de correlación a través del Coeficiente de Correlación de Spearman. Los tres instrumentos resultaron adaptados a nuestra realidad, usando para obtener la validez el método del Item-test y para obtener confiabilidad el Alfa de Crombach (anexo). La prueba de normalidad de Kolmogorov-Smirnov nos indicó que los puntajes de las tres variables no se distribuyeron normalmente por lo que se deben aplicar pruebas no paramétricas. 


\section{RESULTADOS}

La tabla 1 revela hay correlaciones significativas y directas entre desregulación emocional, rumiación e ideación suicida en la muestra estudiada.

\section{Tabla 1}

Correlaciones entre desregulación emocional, rumiacion e ideación suicida en estudiantes que cursan estudios generales en una universidad pública de Lima

\begin{tabular}{|c|c|c|c|c|c|}
\hline & & & $\begin{array}{c}\text { Desregulación } \\
\text { emocional }\end{array}$ & Rumiación & $\begin{array}{l}\text { Ideación } \\
\text { suicida }\end{array}$ \\
\hline \multirow{9}{*}{$\begin{array}{l}\text { Rho de } \\
\text { Spearman }\end{array}$} & \multirow{3}{*}{$\begin{array}{l}\text { Desregulación } \\
\text { emocional }\end{array}$} & Coeficiente de correlación & 1,000 &, $525^{* *}$ & $300^{*}$ \\
\hline & & Sig. (bilateral) & . &, 000 & 000 \\
\hline & & $\mathrm{N}$ & 1330 & 1330 & 1330 \\
\hline & \multirow{3}{*}{ Rumiación } & Coeficiente de correlación &, $525^{\text {** }}$ & 1,000 & ,356 \\
\hline & & Sig. (bilateral) & 000 & . & ,000, \\
\hline & & $\mathrm{N}$ & 1330 & 1330 & 1330 \\
\hline & \multirow{3}{*}{$\begin{array}{l}\text { Ideación } \\
\text { suicida }\end{array}$} & Coeficiente de correlación &, $300^{* * *}$ &, $356^{* *}$ & 1,000 \\
\hline & & Sig. (bilateral) &, 000 &, 000 & \\
\hline & & $\mathrm{N}$ & 1330 & 1330 & 1330 \\
\hline
\end{tabular}

**. La correlación es significativa al nivel 0,01 (bilateral).

El tamaño del efecto de estas correlaciones, tomando en cuenta la propuesta de Cohen (1988), seria grande entre desregulación emocional y rumiación, mediano para las correlaciones entre desregulación emocional e ideación suicida, así como entre rumiacion e ideación suicida.

Como puede observarse, a partir de la tabla 2 , no hay diferencias significativas en la desregulación emocional en función al sexo.

Tabla 2

Comparación de rangos promedios de la desregulación emocional según sexo

\begin{tabular}{llrrr}
\hline & \multicolumn{1}{c}{ Sexo } & N & Rango promedio & \multicolumn{1}{c}{ Suma de rangos } \\
\hline Desregulación emocional & Hombre & 742 & 669,40 & 496691,50 \\
& Mujer & 588 & 660,58 & 388423,50 \\
& Total & 1330 & & \\
U de Mann-Whitney & & & & 215257,500 \\
W de Wilcoxon & & & 388423,500 \\
Z & & &,- 416 \\
Sig. asintót. (bilateral) & & &, 678 \\
\hline
\end{tabular}

a. Variable de agrupación: Sexo

Como puede observarse, a partir de la tabla 3 no hay diferencias significativas en la rumiación en función al sexo. 
Tabla 3

Comparación de rangos promedios de rumiación en relación a sexo

\begin{tabular}{llrrr}
\hline & Sexo & N & Rango promedio & Suma de rangos \\
\hline & Hombre & 742 & 664,42 & 492996,00 \\
Rumiación & Mujer & 588 & 666,87 & 392119,00 \\
& Total & 1330 & \\
U de Mann-Whitney & & & 217343,000 \\
W de Wilcoxon & & & 492996,000 \\
Z & & &,- 116 \\
Sig. asintót. (bilateral) & &, 908 \\
\hline
\end{tabular}

a. Variable de agrupación: Sexo

Como puede observarse, a partir de la tabla 4 , no se han encontrado diferencias significativas en la ideación suicida en función al sexo.

Tabla 4

Comparación de rangos promedios de ideación suicida en relación a sexo

\begin{tabular}{|c|c|c|c|c|}
\hline & Sexo & $\mathbf{N}$ & Rango promedio & Suma de rangos \\
\hline & Hombre & 742 & 677,72 & 502869,50 \\
\hline \multirow[t]{2}{*}{ Ideación suicida } & Mujer & 588 & 650,08 & 382245,50 \\
\hline & Total & 1330 & & \\
\hline U de Mann-Whitney & & & & 209079,500 \\
\hline W de Wilcoxon & & & & 382245,500 \\
\hline Z & & & & $-1,307$ \\
\hline Sig. asintót. (bilateral) & & & & 191 \\
\hline
\end{tabular}

a. Variable de agrupación: Sexo

Como puede observarse, a partir de las tablas 5, no hay diferencias significativas en la desregulación emocional en función a la edad.

Tabla 5

Comparación de rangos promedios de desregulación emocional según edad

\begin{tabular}{llrrr}
\hline & Edad & N & Rango promedio & Suma de rangos \\
\hline \multirow{2}{*}{ Desregulación emocional } & Adolescente & 1133 & 671,63 & 760960,50 \\
& Adulto & 197 & 630,23 & 124154,50 \\
U de Mann-Whitney & Total & 1330 & 104651,500 \\
W de Wilcoxon & & & 124154,500 \\
Z & & & $-1,397$ \\
Sig. asintót. (bilateral) & &, 162 \\
\hline
\end{tabular}

a. Variable de agrupación: Edad 
Como puede observarse, a partir de la tabla 6 , no hay diferencias significativas en la rumiación en función a la edad.

Tabla 6

Comparación entre rangos promedios de rumiacion de acuerdo a edad

\begin{tabular}{llrrr}
\hline & \multicolumn{1}{c}{ Edad } & N & Rango promedio & \multicolumn{1}{c}{ Suma de rangos } \\
\hline & Adolescente & 1133 & 673,47 & 763045,50 \\
Rumiación & Adulto & 197 & 619,64 & 122069,50 \\
& Total & 1330 & & \\
U de Mann-Whitney & & & 102566,500 \\
W de Wilcoxon & & & 122069,500 \\
Z & & & $-1,816$ \\
Sig. asintót. (bilateral) & & &, 069 \\
\hline
\end{tabular}

a. Variable de agrupación: Edad

Como puede observarse, a partir de la tabla 7, si se han encontrado diferencias significativas en la ideación suicida en función a la edad, siendo mayor el rango promedio en los adolescentes.

Tabla 7

Comparación entre rangos promedios de ideación suicida relacionada a edad

\begin{tabular}{llrrr}
\hline & \multicolumn{1}{c}{ Edad } & N & Rango promedio & Suma de rangos \\
\hline \multirow{2}{*}{ Ideación suicida } & Adolescente & 1133 & 675,30 & 765116,00 \\
& Adulto & 197 & 609,13 & 119999,00 \\
& Total & 1330 & & \\
U de Mann-Whitney & & & 100496,000 \\
W de Wilcoxon & & & 119999,000 \\
Z & & & $-2,238$ \\
Sig. asintót. (bilateral) & & &, 025 \\
\hline
\end{tabular}

a. Variable de agrupación: Edad

Como puede observarse, a partir de la tabla 8 si hay diferencias significativas en la desregulación emocional en función al área de formación profesional, observándose que son los ingresantes de humanidades los que muestran mayor desregulación emocional.

Como puede observarse, a partir de la tabla 9, si hay diferencias significativas en la rumiación en función al área de estudios, siendo mayor en humanidades.

Como puede observarse, a partir de la tabla 10, si hay diferencias significativas en la ideación suicida en función al área de estudios, siendo mayor en humanidades. 
Tabla 8

Comparación de rangos promedios de la desregulación emocional según área de formación profesional

\begin{tabular}{llrr}
\hline & Área & N & Rango promedio \\
\hline \multirow{3}{*}{ Desregulación emocional } & Salud & 337 & 619,15 \\
& Básicas & 153 & 578,91 \\
& Ingenierías & 353 & 645,57 \\
& Económicas y de la Gestión & 204 & 574,52 \\
& Humanidades & 283 & 857,95 \\
& Total & 1330 & \\
Chi-cuadrado & & & 96,165 \\
gl & & & 4 \\
Sig. asintót. & & &, 000 \\
\hline
\end{tabular}

a. Prueba de Kruskal-Wallis

b. Variable de agrupación: Área

Tabla 9

Comparación de rangos promedios de la rumiacion según área de formación profesional

\begin{tabular}{llrr}
\hline & Área & N & Rango promedio \\
\hline \multirow{3}{*}{ Rumiacion } & Salud & 337 & 660,06 \\
& Básicas & 153 & 661,12 \\
& Ingenierías & 353 & 673,20 \\
& Económicas y de la Gestión & 204 & 593,06 \\
& Humanidades & 283 & 716,96 \\
& Total & 1330 & \\
Chi-cuadrado & & & 12,573 \\
gl & & & 4 \\
Sig. asintót. & & &, 014 \\
\hline
\end{tabular}

a. Prueba de Kruskal-Wallis

b. Variable de agrupación: Área

\section{Tabla 10}

Comparación entre rangos promedios de ideación suicida acorde al área de formación profesional

\begin{tabular}{|c|c|c|c|c|}
\hline & Área & & $\mathbf{N}$ & Rango promedio \\
\hline & Salud & & 337 & 591,05 \\
\hline \multirow[t]{5}{*}{ Ideación suicida } & Básicas & & 153 & 658,44 \\
\hline & Ingenierías & & 353 & 700,51 \\
\hline & Económicas y de la Gestión & & 204 & 636,72 \\
\hline & Humanidades & & 283 & 735,05 \\
\hline & Total & & 1330 & \\
\hline Chi-cuadrado & & 26,212 & & \\
\hline $\mathrm{gl}$ & & 4 & & \\
\hline Sig. asintót. & & 000 & & \\
\hline
\end{tabular}

a. Prueba de Kruskal-Wallis

b. Variable de agrupación: Área 


\section{DISCUSIÓN}

La hipótesis general del estudio ha sido confirmada, al constatar hay correlaciones significativas y directas entre desregulación emocional, rumiación e ideación suicida en la muestra estudiada. La correlación más fuerte y con un tamaño del efecto grande se da entre desregulación emocional y rumiación, por lo que cabe inferir cierto emparentamiento entre los constructos de desregulación y evitación experiencial. Como queda claro en los estudios internacionales llevados a cabo (Moulds, Kandris, Starr, \& Wong, 2007), la evitación también correlaciona con la rumiación con un tamaño del efecto medio, y es posible que esto sea porque tanto la desregulación como la evitación implican descontrol de respuestas emotivas en situaciones extremas. Por lo tanto, podría ser materia de otro estudio la relación entre ambas.

En cambio, las condiciones de sexo y edad no parecen sensibles en cuanto a influencia sobre la desregulación emocional y la rumiación. Esto señala una uniformidad en cuanto a las características de la presencia de tales variables en la muestre analizada.

Por lo que respecta a la ideación suicida tampoco hay diferencias en la condición sexo, pero sí se encuentra en general un mayor rango de aparición en la edad adolescente. Como señalan Serrano y Flores (2005), la ideación suicida en adolescentes, una edad muy fantasiosa e impulsiva, está ligada con mucha frecuencia a problemas con la pareja.

Un resultado muy interesante es el que se refiere a las diferencias encontradas en las áreas de formación profesional. Allí se ve que tanto la desregulación emocional como la rumiación están significativamente más presentes entre los estudiantes del área de humanidades, por lo que el perfil de los jóvenes que postulan a carreras de dicha área podría estar más ligado que otros a dificultades en relación con sus estados emocionales (sentimientos de ansiedad e inseguridad) y el afrontamiento de sus problemas, y cierta vulnerabilidad a la depresión.

Esto último da pie para sugerir el uso de técnicas psicológicas novedosas con fines preventivos sobre la base útil de tales resultados. Vela (2017), por ejemplo, estudió el tema de la efectividad del mindfulness o "atención plena" para reducir la ideación suicida en jóvenes universitarios víctimas de acoso escolar en España, encontrando una disminución significativa en la ideación suicida y un aumento significativo en la capacidad de mindfulness en el grupo experimental. No estaría mal plantear un programa de intervención basado en mindfulness, adaptado a nuestra realidad universitaria, para reducir los peligros mencionados.

\section{CONCLUSIONES}

Los estudiantes con mayor desregulación emocional presentan mayor rumiación e ideación suicida. 
No hay diferencias significativas en la desregulación emocional, en la rumiación ni en la ideación suicida en función al sexo.

No hay diferencias significativas en la desregulación emocional ni en la rumiación en función a la edad.

Hay diferencias significativas en la ideación suicida en función a la edad, siendo mayor el rango promedio en los adolescentes.

Hay diferencias significativas en la desregulación emocional en función al área de formación profesional, observándose que son los ingresantes de humanidades los que muestran mayor desregulación emocional.

Hay diferencias significativas en la rumiación y en la ideación suicida en función al área de estudios, siendo mayor en humanidades.

\section{Agradecimientos / Acknowledgments:}

Los autores agradecen el apoyo brindado por los las autoridades de la Universdad Nacional Mayor de San Marcos.

\section{Fuentes de financiamiento / Funding:}

Financiado por la Universidad Nacional Mayor de San Marcos. Proyecto con código A18180661, aprobado con Resolución Rectoral 03202-R-18

\section{Rol de los autores / Authors Roles:}

Ejemplo

CVC: Responsables de la investigación.

AGM: Corresponsable de la investigación, análisis de datos.

VML: Responsable de los antecedentes

WMU: Responsable del marco teórico

KEV: Responsable de aplicación de instrumentos

\section{Aspectos éticos / legales; Ethics / legals:}

Los autores declaran no haber incurrido en aspectos antiéticos respetando los códigos de investigación con humanos, se solicitó la autorización respectiva y los participantes firmaron el consentimiento informado el cual brindaba datos de la naturaleza, medios y objetivos de la investigación.

\section{Conflicto de intereses / Competing interests:}

Los autores no ha tenido conflicto de intereses al realizar esta investigación y articulo. 


\section{RECOMENDACIONES}

Sobre la base de los resultados obtenidos en la presente investigación, recomendamos entre otras:

Se indague las relaciones entre la desregulación emocional y la evitación experiencial.

Se elabore un programa de aplicación de técnicas psicológicas para prevenir la presencia significativa de ideaciones suicidas y rumiación en estudiantes universitarios de las áreas más vulnerables

Es importante se profundicen estos estudios relacionando la desregulación emocional, rumiación e ideación suicida con otras variables como pueden ser sectores sociales, sectores laborales, comparar universidades privadas y públicas, y pertenencia o no a tercio superior.

Sería importante que se estudien sectores de edad y sexo ocupados y desocupados, en el caso de edad ver si son jubilados y cuentan con apoyo afectivo familiar.

Es importante profundizar en las características de los jóvenes de humanidades para detectar que aspectos pueden ser desencadenantes de la rumiación e ideación suicida.

\section{REFERENCIAS}

Bandura, A. (2002). Social cognitive theory in cultural context. Journal of Applied Psychology: An International Review, 51, 269-290. http://dx.doi.org/10.1111/14640597.00092 .

Barraca, J. y Pérez, M. (2015). Activación conductual para el tratamiento de la depresión. Madrid: Síntesis.

Beck, A. (2005). Terapia cognitiva para los trastornos de personalidad. Madrid: Paidós Ibérica.

Berghoff, C., Pomerantz, A., Pettibone, J., Segrist, D. \& Bedwell, D. (2012). The Relationship Between Experiential Avoidance and Impulsiveness in a Nonclinical Sample. Behaviour Change. 29. 25-35. http://dx.doi.org/10.1017/bec.2012.6

Cohen, J. (1988). Statistical power analysis for the behavioral sciences (2 ed.). Hillsdale, NJ: Lawrence Erlbaum.

Gliatto, M.F. \& Rai, A.K. (1999). Evaluation and Treatment of Patients with Suicidal Ideation. American Family Physician, 59(6), 1500-1506.

Grajeda, A. (2018). Construcción y validación de un test de aptitud funcional selectora visual en estudiantes de escuelas estatales del primer grado de primaria del distrito de Jesús María, 2018. (Tesis doctoral). Recuperado de http://repositorio.ucv.edu.pe/ bitstream/handle/UCV/21249/Grajeda_MAT.pdf? sequence=1\&isAllowed=y 
Gratz, K. \& Roemer, L. (2004). Multidimensional Assesment of Emotion Regulation and Dysregulation: Development, Factor Structure, and Initial Validation of the Difficulties in Emotion Regulation Scale. Journal of Psychopathology and Behavioral Assesment, 26(1), 41-55.

Hayes, S.C. \& Blackledge, J.T. (2013). Por qué el lenguaje conduce al sufrimiento. En S.C. Hayes y S. Smith (Coords.). Sal de tu mente, entra en tu vida (pp. 53-83). Bilbao: Descleé de Brouwer.

Hayes, S.C., Villatte, M., Levin, M. \& Hildebrandt, M. (2011). Open, aware and active: Contextual approaches as an emerging trend in the behavioral and cognitive therapies. Annual Review of Clinical Psychology, 7, 141-168. http://dx.doi.org/10.1146/annurevclinpsy-032210-104449

Hayes, S., Levin, M. Plumb-Vilardaga, J., Villatte, J. \& Pistorello, J. (2013). Acceptance and commitment therapy and contextual behavioral science: Examining the progress of a distinctive model of behavioral and cognitive therapy. Behavior Therapy, 44, 180-198. http://dx.doi.org/10.1016/j.beth.2009.08.002.

Hayes, S.C., Strosahl, K.D. \& Wilson, K.G. (2014). Terapia de Aceptación y Compromiso. Proceso y práctica del cambio consciente (Mindfulness). Bilbao: Desclée De Brouwer.

Hervás, G.; Vázquez, C., (2006). Explorando el origen emocional de las respuestas rumiativas: el papel de la complejidad emocional y la Inteligencia Emocional, Ansiedad y estrés, 12(2-3), 279-292.

Hervás G. \& Jódar, R. (2008). Adaptación al castellano de la Escala de Dificultades en la Regulación Emocional. Clínica y Salud, 19, 139-156.

Hervás, G. (2008). Adaptación al castellano de un instrumento para evaluar el estilo rumiativo: la escala de respuestas rumiativas. Revista de Psicopatología y Psicología Clínica, 13(2), 111-121. Recuperado de http://www.aepcp.net/arc/(4)\%202008(2). Herv\%C3\%A1s.pdf

Inostroza I. (2016). Procesos psicológicos de riesgo de intento suicida en usuarios de servicios de salud mental de nivel especializado de la Provincia de Concepción Tesis para optar al grado de Doctor en Psicología. Universidad de Concepción Dirección de Postgrado Facultad de Ciencias Sociales-Programa de Doctorado en Psicología. Concepción, Chile.

Lazarus, R.S. (1999). Estrés y emoción: manejo e implicaciones en nuestra salud. Bilbao: Desclée de Brouwer.

Linehan, M. (2003) Manual de tratamiento de los trastornos de personalidad limite. Barcelona: Paidós.

Moulds, M., Kandris, E., Starr, S., \& Wong, Ch.. (2007). The relationship between rumination, avoidance and depression in a non-clinical sample. Behaviour research and therapy. 45. 251-61. 10.1016/j.brat.2006.03.003.

Nicolai, K. A. (2015). Rumination and Impulsivity as Moderators of the Relationship between Negative Affectivity and Nonsuicidal Self-Injury among College Students(Ph.D. Thesis). Retrieved from Dissertation Abstracts International. 
Nolen-Hoeksema, S. (1991). Responses to Depression and Their Effects on the Duration of Depressive Episodes. Journal of Abnormal Psychology, 100(4), 569-582. doi:10.1037/0021-843X.100.4.569

Organización Mundial de la Salud (2003). Informe mundial sobre la Violencia y la Salud. Recuperado de la página de internet del organismo: http://www.who.int/whr/2003/es/

Organización Mundial de la Salud (2015). Informe mundial. Recuperado de la página de internet del organismo: http://www.who.int/gho/publications/es/

Pérez-Álvarez, M. (2014). Las terapias de tercera generación como terapias contextuales. Madrid: Síntesis.

Rosales, J. C., Córdova, M. y Ramos, R. (2012). Ideación suicida en estudiantes mexicanos: un modelo de relación múltiple con variables de identificación personal. Psicología y Salud, 22(1), 63-74. doi.org/10.25009/pys.v22i1.558

Sánchez, H. y Reyes, C. (2015). Metodología y diseños en la investigación científica. Lima: Universidad Ricardo Palma.

Shaurya, R., Whitmoyer, P., Aldao, A. \& Schirda, B. (2017) Mindfulness and emotion regulation in older and young adults, Aging \& Mental Health, 21:1, 77-87, DOI: 10.1080/13607863.2015.1100158

Schore, A., (2003). Affect dysregulation and disorders of the self. New York: Norton.

Serrano G. y Flores M. (2005) Estrés, respuestas de afrontamiento e ideación suicida en adolescentes. Revista Psicología y Salud, Vol. 15, Núm. 2: 221-230.

Seligowski, A. \& Orcutt, H. (2015). Examining the Structure of Emotion Regulation: A Factor-Analytic Approach. Journal of Clinical Psychology, 71, 1004-1022. doi. org/10.1002/jclp.22197

Suarez, Y.P; Restrepo D., Caballero D, Carmen C. (2016). Ideación suicida y su relación con la inteligencia emocional en universitarios colombianos. Rev. Univ. Ind. Santander. Salud, 48(4), 470-478. doi.org/10.18273/revsal.v48n4-2016005.

Vela M. (2017). Efectividad del mindfulness en reducir ideación suicida en jóvenes universitarios víctimas de acoso escolar. Tesis para optar el Título de Psicóloga Clínica. Colegio de Ciencias Sociales y Humanidades Universidad San Francisco de Quito.

Velásquez Centeno, C., Grajeda Montalvo, A., Montero López, V., Montgomery Urday, W., \& Egusquiza Vásquez, K. (2018). Evitación experiencial, rumiación e impulsividad en estudiantes de la Universidad Nacional Mayor de San Marcos. Revista De Investigación En Psicología, 21(1), 15-26. https://doi.org/10.15381/rinvp.v21i1.15110

Villalobos, F.H. (2009). Ideación suicida en jóvenes: formulación y validación del modelo integrador explicativo en estudiantes de educación secundaria y superior. Tesis Doctoral. Universidad de Granada.

Villalobos, F.H. (2010). Validez y fiabilidad del Inventario de Ideación Suicida Positiva y Negativa-PANSI, en estudiantes colombianos. Universitas Psychologica, 9(2),509520. http://dx.doi.org/10.11144/Javeriana.upsy9-2.vfii 